\title{
A Delta-Star-Based Multipoint Fiber Bragg Grating Sensor Network
}

\author{
Shu-Tsung Kuo, Peng-Chun Peng, Jen-Wei Sun, and Ming-Seng Kao, Member, IEEE
}

\begin{abstract}
This work presents a novel delta-star architecture for large-scale multipoint fiber sensor networks with enhanced reconfigurable functionality. Unlike conventional star networks, the proposed network is divided into several delta regions, each containing only one feeder fiber. This delta-star structure significantly reduces the complexity and implementation cost of conventional star networks. A concatenated dual-ring structure with the self-healing function is designed to overcome the star's weakness in reliability. Numerous sensor ring subnets (SRSNs) are utilized to serve a large number of fiber Bragg grating sensors. Delta regions are partitioned upon the criterion of inter-region traffic uniformization; this simplifies the interrogation process and time slot assignment. The reconfigurable function for this multipoint sensor network, which is based on the optical switching technique, is utilized to ensure service continuity when fibers fail. The protection mechanism is illustrated with several multiple link failure cases. Finally, an experimental network is implemented to demonstrate the feasibility and survivability of the proposed sensor network.
\end{abstract}

Index Terms-Delta-star network, fiber Bragg grating (FBG), fiber ring laser, fiber sensor network, network partitioning, reconfigurable function.

\section{INTRODUCTION}

A fiber Bragg grating (FBG) is a simple and low-cost wavelength-selective element with EMI immunity and large-scale multiplexing capabilities. Moreover, the strain or temperature drift on an FBG typically leads to its resonant Bragg wavelength shift. These characteristics render FBGs suitable for large-scale multipoint structural sensing measurements. The feasibility of FBGs as sensing elements has been demonstrated experimentally with several large-scale FBG sensor networks [1]-[5]. As the amount of sensing information to be handled by a sensor network is increasing, more and more attention has focused on the network protection ability

Manuscript received February 04, 2010; accepted August 26, 2010. Date of publication September 27, 2010; date of current version February 04, 2011. This work was supported by the National Science Council of the Republic of China, Taiwan, under Contract NSC 98-2221-E-027-007-MY3. The associate editor coordinating the review of this paper and approving it for publication was Prof. Evgeny Katz.

S. T. Kuo is with the Department of Electrical Engineering, National ChiaoTung University, Hsinchu 300, Taiwan (e-mail: rogerkuo13@gmail.com).

P. C. Peng is with the Department of Electro-Optical Engineering, National Taipei University of Technology, Taipei 106, Taiwan (e-mail: pengpc@gmail. com).

J. W. Sun is with the Department of Electrical Engineering, National Chi Nan University, Nantou County 54561, Taiwan.

M. S. Kao is with the Department of Electrical Engineering, National ChiaoTung University, Hsinchu 300, Taiwan (e-mail: mskao@cc.nctu.edu.tw).

Color versions of one or more of the figures in this paper are available online at http://ieeexplore.ieee.org.

Digital Object Identifier 10.1109/JSEN.2010.2076278 and survivability [6]-[9]. This generates new challenges for the topology design and the reconfigurable function when devising a multipoint sensor network. A large-scale FBG sensor network constructed simply on an in-line, star, or tree topology generally lacks the sufficient protection capability against fiber failure [10]-[13]. To enhance network reliability, a complementary star-ring FBG sensor network architecture was developed [14]; however, implementing a large-scale fiber sensor network with a regular star is both costly and difficult.

This work proposes a novel architecture for large-scale multipoint FBG sensor networks with powerful reconfigurable functionality. A delta-star architecture is designed to markedly reduce the difficulty and expense in implementing a large-scale fiber sensor network. The network is divided into several delta regions with each having near-uniform sensing traffic load to simplify the interrogation process and time slot assignment. Moreover, spatial wavelength reuse and time-division multiplexing (TDM) signal deliveries for separate sensor ring subnet (SRSN) clusters are carried out in the sensor network, which can unify the optical components used in different delta regions. Although the star feeder fibers are sparsely deployed, the performance of the proposed delta-star sensor network, being superior to that of a conventional one, is achieved by the well-designed reconfigurable function. Unlike the hybrid star-ring structure in [14], the proposed architecture offers a low-cost and flexible infrastructure for multiplexing optical fiber sensors.

The reconfigurable function to prevent our sensor network from a severe sensing signal loss in case of fiber failure(s) is supported by optical switches. In the network, however, the use of these optical switches introduces additional signal losses, further reducing its average signal-to-noise ratio (SNR). To improve this drawback, a tunable fiber ring laser with each installed FBG as its lasing cavity mirror is used in the proposed sensor network to provide the intense lasing power. The proposed delta-star sensor network with the reconfigurable function can be embedded in such structures as dams, bridges, tunnels, and buildings for their health monitoring.

The remainder of this paper is organized as follows. Section II describes the network design in detail. Next, for illustrative purposes, several multiple link failure cases are presented in Section III to explain how our FBG sensor network works in normal and protection conditions. In Section IV, an experimental network is implemented to demonstrate the feasibility and reliability of the proposed network. Finally, Section V gives conclusions. 


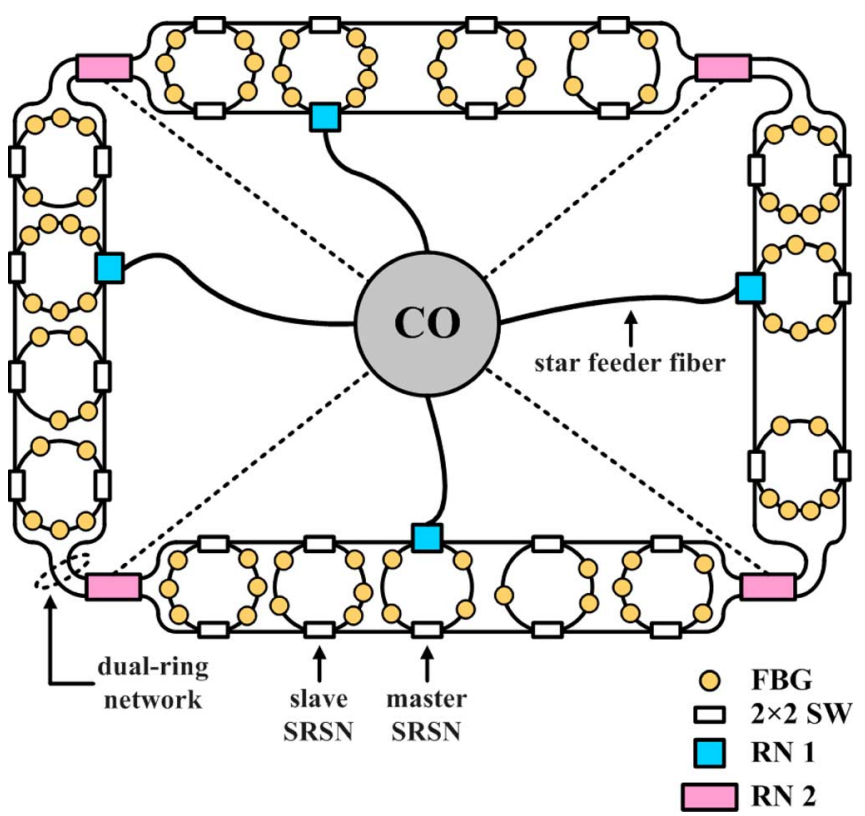

Fig. 1. Schematic diagram of the proposed delta-star architecture for a multipoint FBG sensor network. (CO: central office, FBG: fiber Bragg grating, $2 \times 2$ SW: $2 \times 2$ optical switch, RN 1/2: remote node 1/2, master/slave SRSN: master/slave sensor ring subnet).

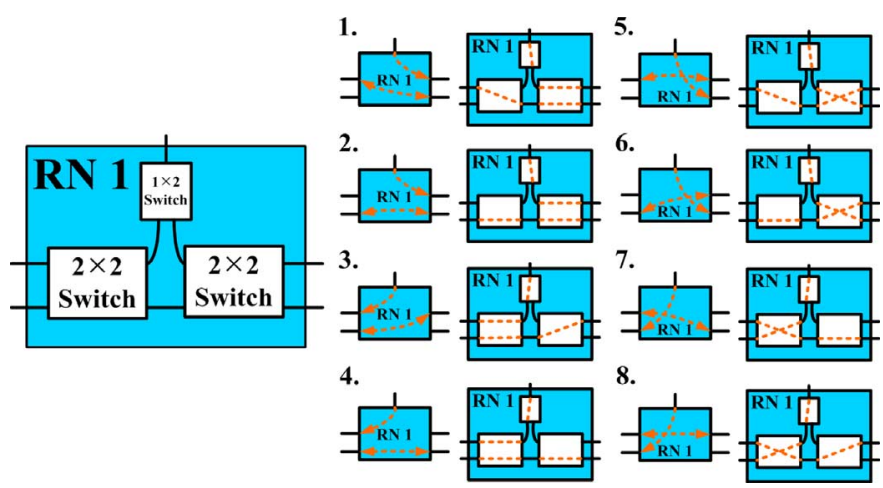

Fig. 2. Configuration of RN 1 and all its possible switching states.

\section{Network ARChiteCtURE AND DESIGN}

\section{A. Network Architecture}

The multipoint smart sensor network based on the proposed delta-star architecture is illustrated in Fig. 1. As shown in this figure, the network is partitioned into several delta regions, where each region defined by two adjacent dashed lines contains many SRSNs. Within a delta region, moreover, a star fiber link is served as the bridge between the $\mathrm{CO}$ and its corresponding SRSN cluster. The particular SRSN linked directly to the $\mathrm{CO}$ via the remote node 1 (RN 1) is called the master SRSN and the others are the slave SRSNs.

The CO is responsible for the supply of the light source and the discrimination of sensing signals reflected from the sensor network. In our design, the RN 1 consists of a $1 \times 2$ and two $2 \times 2$ optical switches, as depicted in Fig. 2, wherein all its possible switching states are demonstrated as well. For simplicity, the control units of these switches are not shown in the figure. The function of RN 1 is to effectively transfer sensing signals

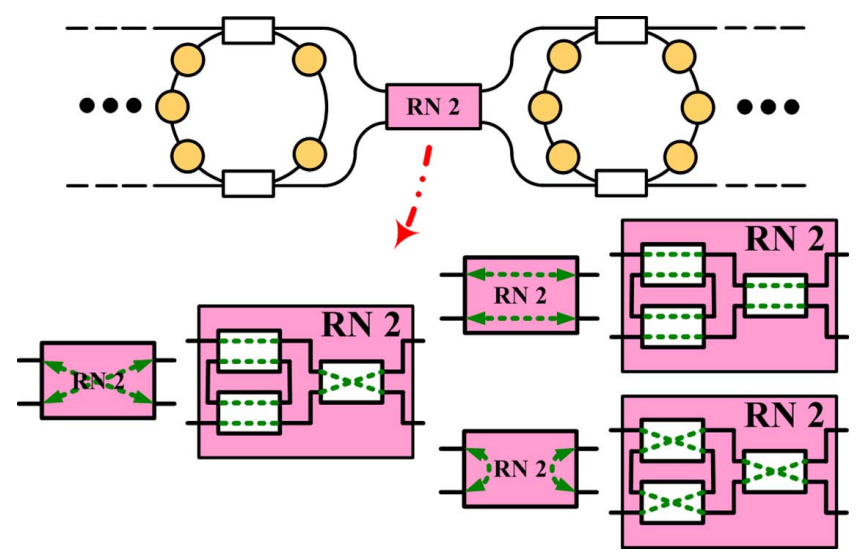

Fig. 3. Switching states of RN 2 composed of three $2 \times 2$ optical switches.

from the CO to the SRSNs. In addition, all the SRSNs inside a delta region are connected with a dual-ring network and any two adjacent SRSN clusters are linked by a remote node 2 (RN 2), as we can see in Fig. 3. Meanwhile, the three switching states of the RN 2 are presented in this figure. The state of any optical switch in RN 1 or RN 2 is controlled by the TDM signal, and it would be employed to support the reconfigurable function in case of fiber failure(s) if necessary. In normal operation, the sensing signals for an SRSN are sent from/to the CO through the star feeder fiber in the same delta region, and in protection operation, they are delivered via the star fiber in the same or neighboring region upon the fiber failure scenario occurred, which will be illustrated later.

Because the sensing traffic load inside each partition is unified as far as possible in our design, the amount of FBG sensors included in each delta region is almost equal in the proposed network. However, since the number of FBG sensors installed in each ring subnet is varied, the number of SRSNs contained in each delta region may be different.

\section{B. Network Design}

Different from conventional star sensor networks, we divide our network into several delta regions in accordance with practical circumstances, where only one star feeder fiber is installed in each partition, as illustrated in Fig. 1. Moreover, the sensing traffic load in each partition is allocated as uniformly as possible, which can simplify the interrogation process and time slot assignment. The design method of the proposed sensor network is addressed as follows [15].

Let $N$ be the total number of SRSNs in the network and $K$ be the number of delta regions to be partitioned. Assume $b_{i}$ is the number of FBG sensors in the $i$ th SRSN, $i=1,2, \ldots, N$, then the total number of FBG sensors in the whole network is given as

$$
B=\sum_{i=1}^{N} b_{i} .
$$

Thus, the average number of FBG sensors per delta region is approximated as

$$
\Lambda=\left\lfloor\frac{B}{K}\right\rfloor
$$


where " $\lfloor\rho\rfloor$ " represents the maximum integer equal to or less than a real number $\rho$.

Let $\Lambda_{j}, j=1,2, \ldots, K$, denote the number of FBGs inside the $j$ th delta region. We define the percentage of absolute variation margin admitted on $\Lambda_{j}$ with respect to $\Lambda$ is $\tau_{\Lambda}$. Therefore, the minimum allowable value of $\Lambda_{j}$ is

$$
\left(\Lambda_{j}\right)_{\min }=\left\lceil\Lambda\left(1-\frac{\tau_{\Lambda}}{100}\right)\right\rceil \triangleq \Phi_{\min }
$$

where " $\lceil\rho\rceil$ " represents the minimum integer equal to or greater than a real number $\rho$, and the maximum of $\Lambda_{j}$ is

$$
\left(\Lambda_{j}\right)_{\max }=\left\lfloor\Lambda\left(1+\frac{\tau_{\Lambda}}{100}\right)\right\rfloor \triangleq \Phi_{\max } .
$$

Note that a relatively small $\tau_{\Lambda}$ will lead to a more uniform traffic distribution among different delta regions; however, it may make the partitioning algorithm get into traps.

1) Phase I: Partition of K Delta Regions: The first phase of the algorithm is to divide the $N$ SRSNs into $K$ clusters, where each cluster has around $\Lambda$ FBGs inside. Let $S_{i}, i=1,2, \ldots, N$, denote the $N$ SRSNs to be partitioned. We start the work with $S_{1}$ which is served as the leading SRSN of the unformed first cluster. Then, we get $S_{1}$ and its $m$ neighboring SRSNs (i.e., $\left.S_{2}, S_{3}, \ldots, S_{m+1}\right)$ together to be a cluster, such that the total number of FBGs inside this cluster, denoted as $\Lambda_{1}$, is within $\left[\Phi_{\min }, \Phi_{\max }\right]$. Namely

$$
\Phi_{\min } \leq \Lambda_{1}=\sum_{i=1}^{m+1} b_{i} \leq \Phi_{\max } .
$$

If the specification of (5) cannot be met when we start with $S_{1}$, another leading SRSN is chosen and the above process is repeated. After the first cluster is formed, an SRSN not included in and subsequent to the first cluster is picked as the leading SRSN for the second cluster and the above clustering process is repeated. As soon as $\Lambda_{j}$ of any SRSN cluster fails to fill the bill, we divide the $N$ SRSNs anew. The process will be continued until all the $K$ clusters are formed. Note that if the admitted variation margin $\tau_{\Lambda}$ is too small, it may happen that the $K$ clusters cannot be successfully formed. Under this condition, a larger $\tau_{\Lambda}$ is chosen and the partitioning process is repeated. When the partitioning process is completed, we get

$$
\Phi_{\min } \leq \Lambda_{j} \leq \Phi_{\max } \quad j=1,2, \ldots, K .
$$

It means the number of FBGs inside each of the $K$ clusters is within the allowed variation margin.

2) Phase II: Assignment of Master SRSNs: After the $K$ delta regions are formed, we proceed to assign the master SRSN of each delta region. In our design, the selection of the master SRSN for a delta region is based on a parameter $H_{x}$, and the procedure of master SRSN assignment is explained in the following. For a delta region $x$, there are several candidate master SRSNs inside. First, we pick one of them as the tentative master SRSN for region $x$ and then evaluate the parameter $H_{x} \triangleq$ $\left.\sum_{i}\left(b_{i, x} \times h_{i, x}\right)\right)$ upon it, where $b_{i, x}$ denotes the number of FBG sensors involved in an SRSN $S_{i}$ in region $x$ and $h_{i, x}$ signifies the minimum-hop distance between $S_{i}$ and CO through the local tentative master SRSN. Next, by using the same method,
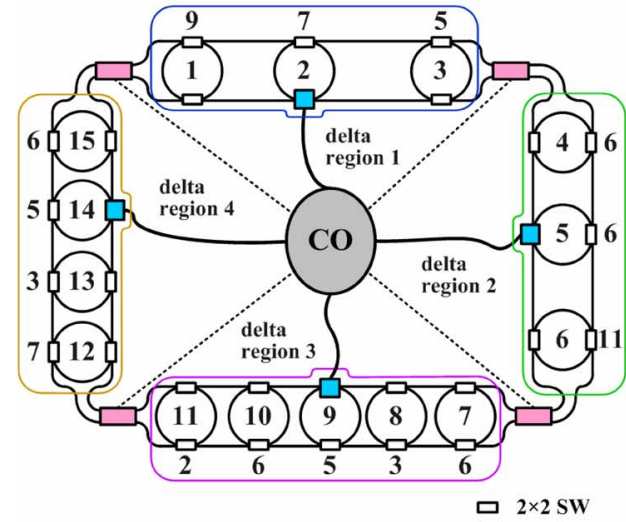

$\square$ RN 1

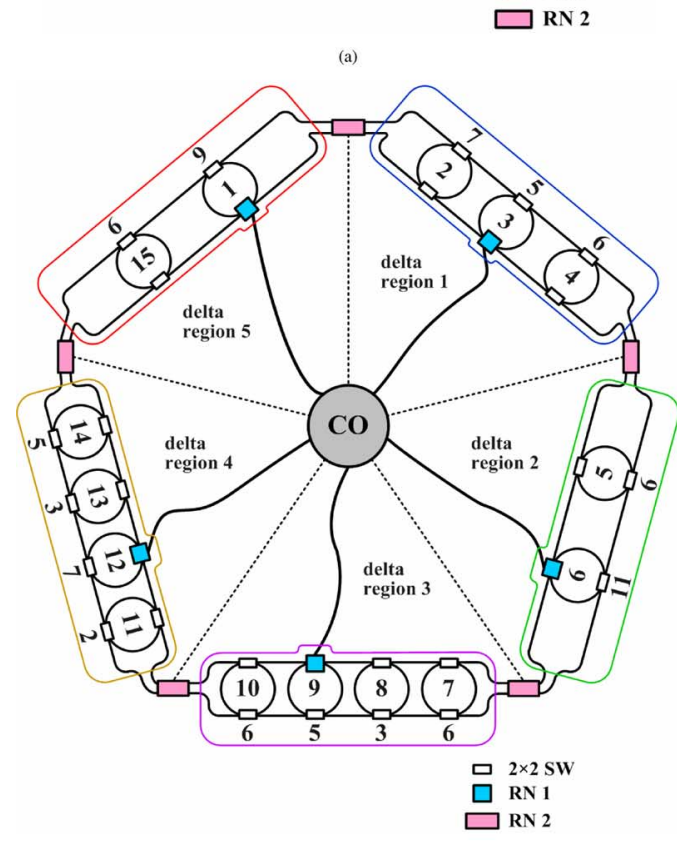

(b)

Fig. 4. A network example under partitioning requests of (a) four delta regions and (b) five delta regions.

the estimation of parameter $H_{x}$, upon each of the other candidate master SRSNs inside this delta region, is received. The specific SRSN with the minimum $H_{x}$ is designated as the master SRSN of region $x$ in our scheme to reduce the mean intra-region retransmission hops in case of internal fiber failures. When the master SRSN of each delta region is assigned, the design is completed.

To gain more insight into our design, a network example under different partitioning requests is provided for illustration, whose component SRSNs are shown in Fig. 4. For simplicity, we employ a circle to represent a lower-level SRSN in this figure, where the numeral inside a circle denotes its serial number and the one beside a circle signifies the number of FBG sensors installed in it. First, the network with four delta regions is designed, where each delta region contains the almost equal number of FBG sensors, as shown in Fig. 4(a). Meanwhile, the local master SRSN for each delta region is specified using the above assignment method. As depicted in the figure, the numbers of FBG sensors in delta regions 1, 2, 3 and 4 are 21, 23, 22 and 21, respectively. When the number of FBG sensors 


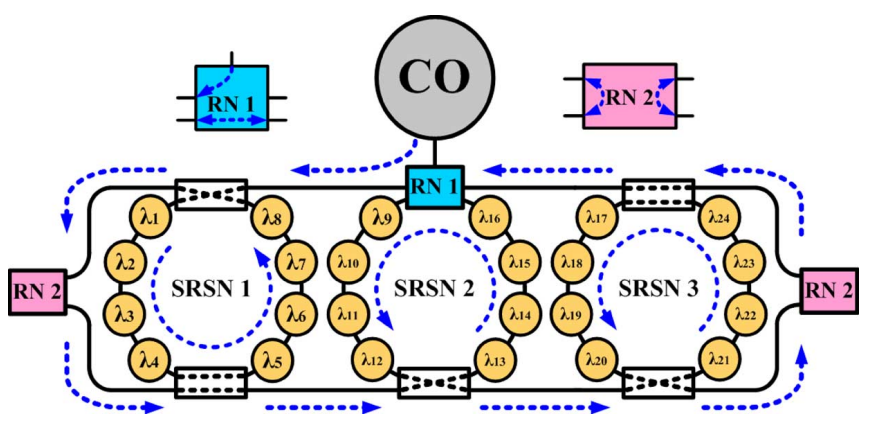

Fig. 5. Illustration of the situation when an SRSN cluster is selected by the corresponding TDM signals in normal operation.

in a delta region increases, the average loss of sensing signals, in general, increases too.

If five delta regions are specified in the above example, the resultant network is shown in Fig. 4(b). In this case, the numbers of FBG sensors in delta regions 1, 2, 3, 4 and 5 are 18, $17,20,17$ and 15 , respectively. When the number of delta regions increases, the amount of FBG sensors in a delta region decreases, thereby leading to a lower average loss of sensing signals. However, the implementation cost and complexity increase too. Therefore, the number of delta regions is a critical factor in practical network design.

\section{NETWORK OPERATION AND RECONFIGURABLE FUNCTION}

In this section, the operations of the proposed FBG sensor network under normal and protection modes are illustrated with several null and multiple link failure scenarios. The spatial wavelength reuse for different SRSN clusters, and the TDM switching and sensing signal deliveries for them are implemented in the sensor network. Fig. 5 schematically depicts the situation when an SRSN cluster is selected by the corresponding TDM signals in normal operation. In this figure, the TDM switching and sensing signals for this SRSN cluster are all sent along the path indicated by dashed lines. Therefore, the FBG sensors installed in its SRSN 1, SRSN 2, and SRSN 3 are successively scanned under the normal mode in our scheme. The TDM technology cooperated with the delta-star structure make the proposed sensor network be able to support many sensor elements. For such a large-scale multipoint sensor network, however, the reconfigurable function to protect it against fiber break(s) is required, and how our reconfigurable function reacts to different fiber failure scenarios is illustrated with two multiple link failure cases in the following.

The first multiple failure case is demonstrated in Fig. 6. First, with the aid of reconfigurable function, the sensing signals for those FBGs in SRSN 2 can be separately retransmitted through Paths 1, 2, 3, and 4, as shown in Fig. 6(a). During that process, the sensing information about the FBGs with $\lambda_{1} \sim \lambda_{4}$ in SRSN 1 and with $\lambda_{21} \sim \lambda_{24}$ in SRSN 3 is obtained as well. Next, we deliver the sensing signals for FBGs with $\lambda_{5}$ and $\lambda_{6}$ in SRSN 1 via Path 5, and then reconfigure the fiber link to sense the states of FBGs with $\lambda_{19}$ and $\lambda_{20}$ through Path 6, as depicted in Fig. 6(b). Finally, by means of the functionality, the sensing information about FBGs with $\lambda_{17} \sim \lambda_{18}$ in SRSN 3 and with $\lambda_{7} \sim \lambda_{8}$ in SRSN 1 can be successively acquired via Paths 7
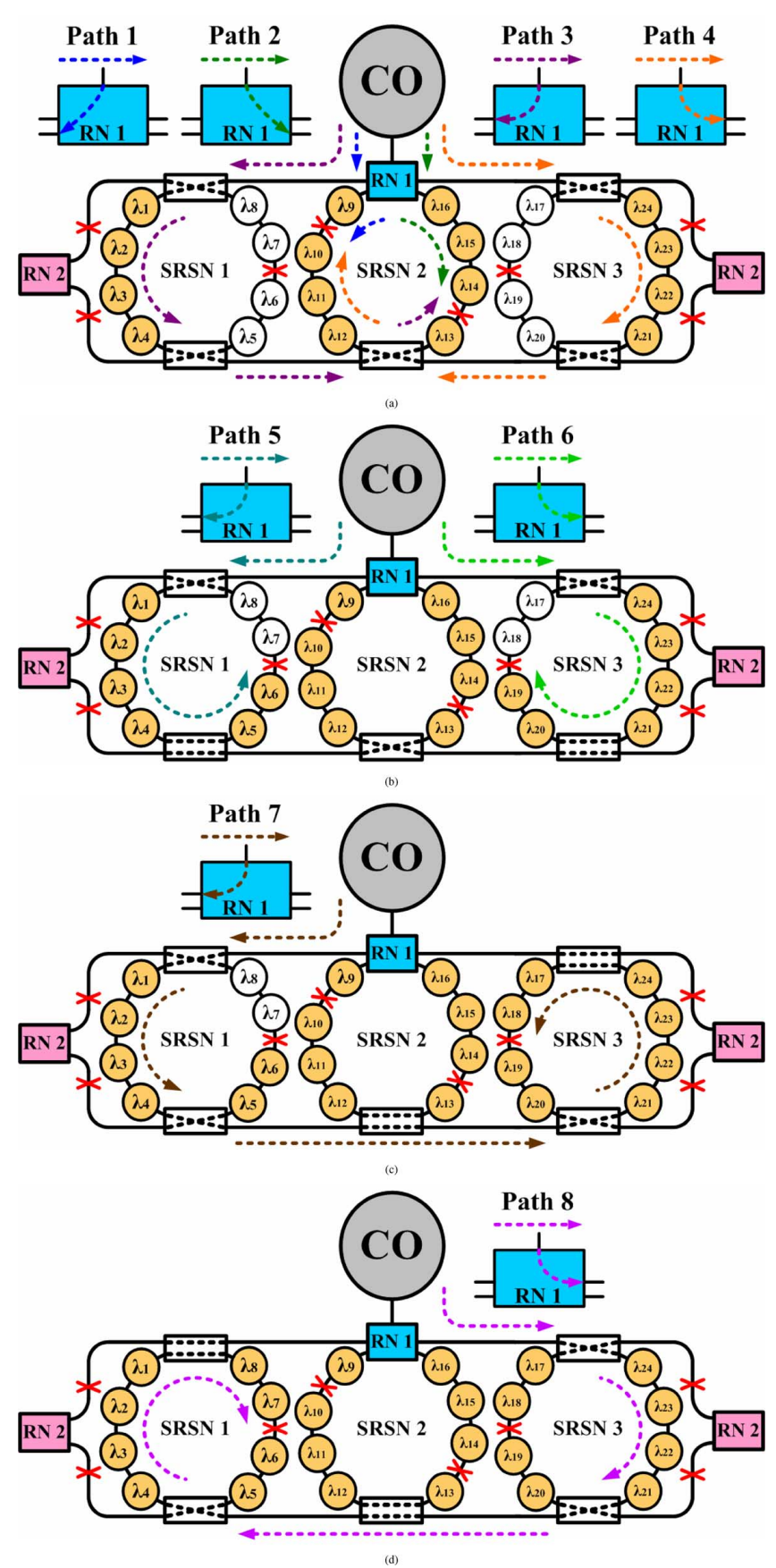

Fig. 6. Retransmissions of sensing signals for FBGs with (a) $\lambda_{n}$ ( $\mathrm{n}=1,2,3$, $4,9,10,11,12,13,14,15,16,21,22,23,24)$, (b) $\lambda_{n}(\mathrm{n}=5,6,19,20)$, (c) $\lambda_{n}$ $(\mathrm{n}=17,18)$, and (d) $\lambda_{n}(\mathrm{n}=7,8)$ in the first network failure case.

and 8, as presented in Fig. 6(c) and (d), respectively. Thus, the reconfigurable function enables the retransmissions of all the lost sensing signals in such a fiber failure scenario.

The second multiple failure case is shown in Fig. 7, wherein the sensing information about SRSNs 1, 2, and 3 is unavailable through the star feeder fiber in the same delta region. However, we can make use of the reconfigurable function to retransmit the sensing signals for them via the star fibers in the neighboring regions, as indicated in this figure. Because many optical switches are used for the reconfigurable function, this induces additional signal losses and further reduces the average 


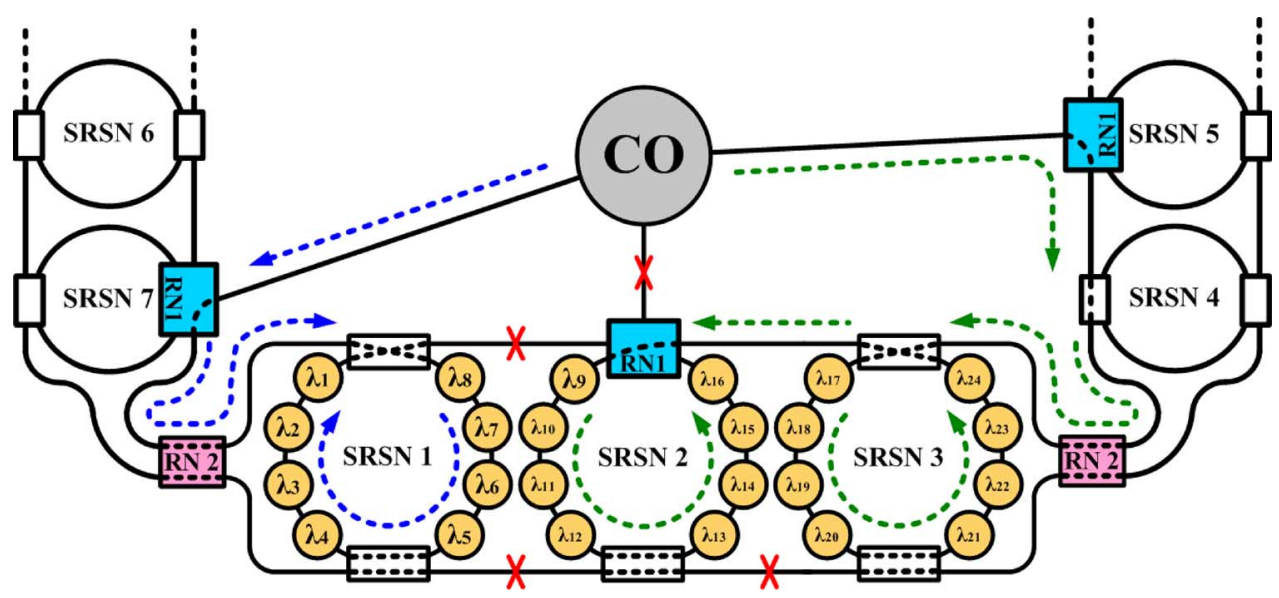

Fig. 7. The given SRSN cluster in the second multiple link failure case. The sensing information about FBGs in its SRSNs 1,2 , and 3 are successively obtained via two different retransmission paths.

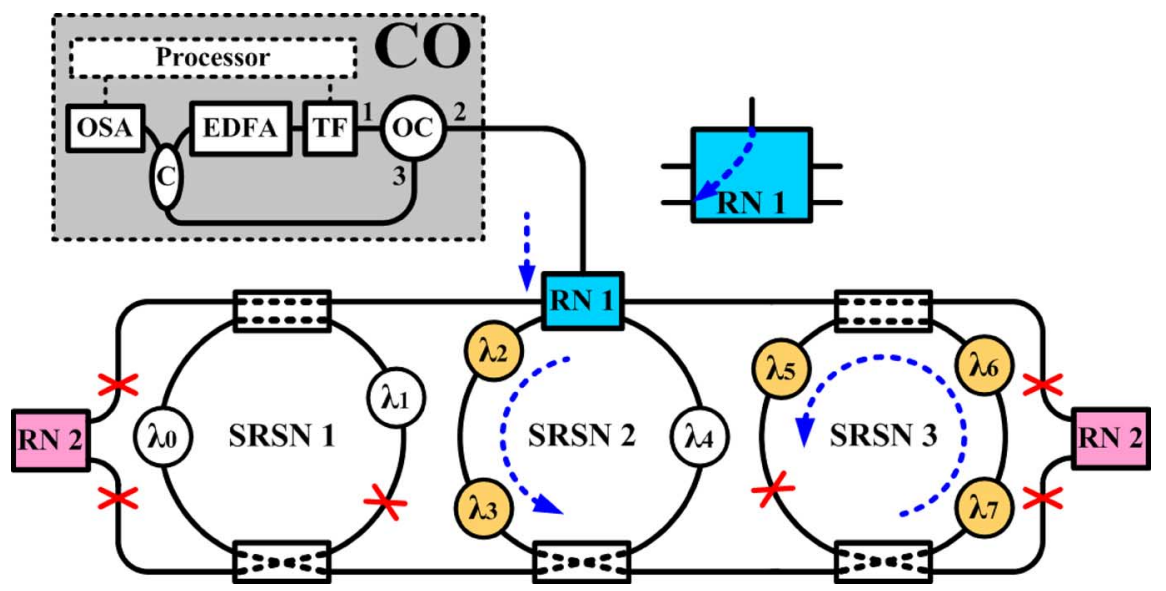

Fig. 8. Experimental setup of one proposed FBG sensor network in a multiple fiber failure case. (OC: optical circulator, TF: tunable bandpass filter, EDFA: erbium-doped fiber amplifier, C: 10/90 fiber coupler, OSA: optical spectrum analyzer). Under the link condition, sensing signals for FBGs with $\lambda_{n}(\mathrm{n}=0,1,4)$ are lost.

SNR of the network. A tunable fiber ring laser configuration with each installed FBG as its lasing cavity mirror is thus applied to our network to improve such a shortcoming, while the effectiveness of this scheme will be experimentally verified in Section IV. Consequently, the delta-star architecture with reconfigurable functionality is suitable for the construction of intelligent and robust multipoint sensor networks.

\section{EXPERIMENTAL SETUP AND RESUltS}

To demonstrate the feasibility and survivability of the proposed architecture, we set up an experimental network, as shown in Fig. 8. Here, three SRSNs of the same cluster are investigated. As depicted in this figure, the $\mathrm{CO}$ is equipped with an optical circulator (OC), a tunable bandpass filter (TF), an erbium-doped fiber amplifier (EDFA), and a 10/90 fiber coupler (C). Each SRSN composed of several FBG sensors is attached to the dualring network via optical switches. The insertion losses of the $1 \times 2$ and the $2 \times 2$ optical switches used are both below $1 \mathrm{~dB}$. In addition, the Bragg wavelengths $\lambda_{0} \sim \lambda_{7}$ of those FBG sensors employed in our experiment are sequentially $1535.94,1538.88$, $1542.09,1544.94,1547.91,1551.03,1553.97$, and $1558.38 \mathrm{~nm}$. In our scheme, the lasing wavelengths of the fiber ring laser in

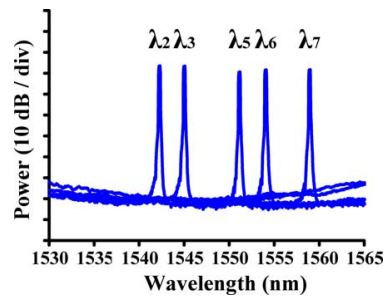

Fig. 9. Output spectra of the fiber ring laser under the link condition shown in Fig. 8.

this network are determined by the TF and those FBG sensors, where each of the FBG sensors is served as a cavity mirror of the fiber ring laser. The 3-dB bandwidth of the TF is around $0.35 \mathrm{~nm}$. When the passband wavelength of the TF is identical to the reflected wavelength from an FBG sensor, the lightwave system lases with sufficient gain. Accordingly, the lasing wavelength of the lightwave system can be used for the measurement of the slight strain or temperature variation on the FBG sensor. The passband wavelength of the TF is tuned with a controller to track the eight Bragg wavelengths $\lambda_{0} \sim \lambda_{7}$ of those deployed FBG sensors. The $10 \%$ of any lasing light is fed into an optical 


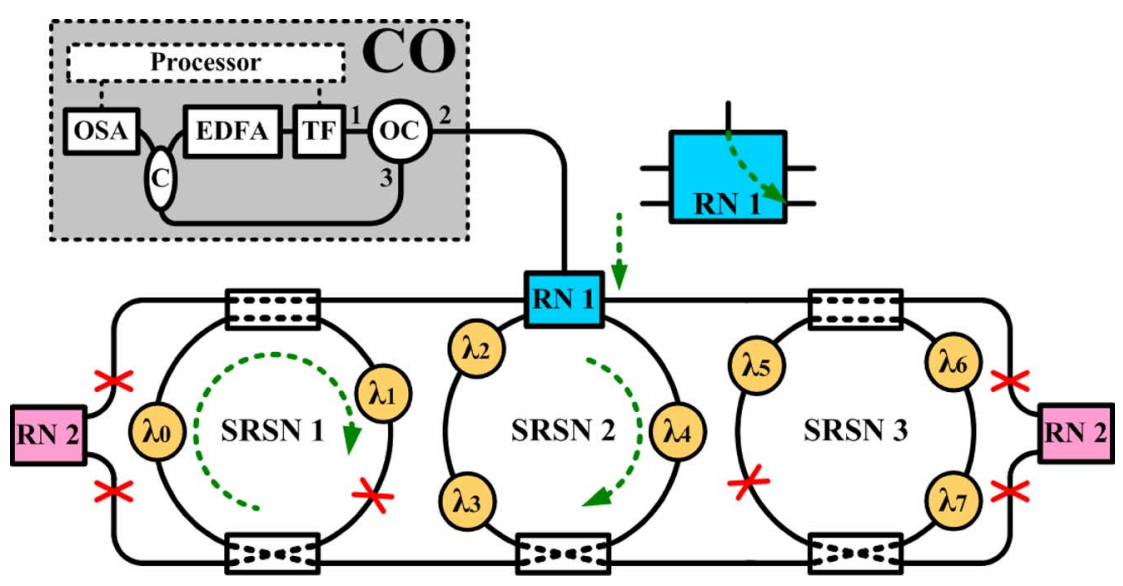

Fig. 10. Reconfiguration of RN 1 leads to the recovery of lost sensing information about FBGs with $\lambda_{n}(\mathrm{n}=0,1,4)$.

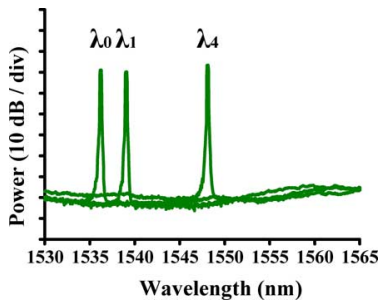

Fig. 11. Output spectra of the fiber ring laser under the link reconstruction shown in Fig. 10.

spectrum analyzer (OSA) via the fiber coupler in the $\mathrm{CO}$ for the discrimination of lasing wavelength.

In the multiple fiber failure scenario, as shown in Fig. 8, the sensing signals for the FBGs with $\lambda_{n}(\mathrm{n}=2,3,5,6,7)$ can be delivered along the path indicated by dashed lines, but those for the FBGs with $\lambda_{n}(\mathrm{n}=0,1,4)$ are lost. The consequent output spectra of the fiber ring laser are demonstrated in Fig. 9. However, we can modify the state of RN 1 to reconfigure the fiber link and retransmit the lost sensing signals for FBGs with $\lambda_{n}$ $(\mathrm{n}=0,1,4)$, as shown in Fig. 10. Meanwhile, Fig. 11 displays the resultant output spectra measured by the OSA. From Figs. 9 and 11, we can observe that the average SNR of the experimental sensor network exceeds $60 \mathrm{~dB}$ due to the intense lasing power of the fiber laser. Therefore, the reconfigurable architecture makes cost-effective, smart and reliable multipoint sensor networks become feasible.

The minimum resolvable wavelength shift that our OSA can measure is $0.05 \mathrm{~nm}$, being corresponding to a minimum resolution of around $41.5 \mu \varepsilon$ for the strain variation [1]. For the TF used in our experiment, moreover, its maximum scanning rate from 1535 to $1565 \mathrm{~nm}$ is about $1 \mathrm{~Hz}$. The system resolution and response time can be improved by using a better wavelength interrogator and faster optical switches [16].

\section{CONCLUSION}

This work develops a novel, highly reliable delta-star architecture for multipoint smart FBG sensor networks. The introduction of delta-star architecture markedly reduces difficulty and expense in network deployment, while the inherent weakness of the star network (i.e., its poor reliability) is overcome by the self-healing function of the concatenated dual-ring network. Many SRSNs are used to serve a large number of FBG sensors in the proposed network. The full path protection capability of this network is achieved by deploying reconfigurable optical switches, such that it can protect numerous FBG sensors against fiber break(s). Finally, retransmissions of sensing signal flows under network link reconstructions have been experimentally demonstrated and characterized. The intense lasing power of the fiber ring laser installed in the experimental sensor network causes an average measured SNR of $>60 \mathrm{~dB}$. The experimental results reveal that, in terms of scalability and reliability, the proposed architecture is a promising candidate for large-scale multipoint FBG sensor networks.

\section{REFERENCES}

[1] A. D. Kersey, M. A. Davis, H. J. Patrick, M. Leblance, K. P. Koo, C. G. Askins, M. A. Putnam, and E. J. Friebele, "Fiber grating sensors," J. Lightw. Technol., vol. 15, pp. 1442-1463, Aug. 1997.

[2] S. Diaz, G. Lasheras, and M. Lopez-Amo, "WDM bi-directional transmission over $35 \mathrm{~km}$ amplified fiber-optic bus network using Raman amplification for optical sensors," Opt. Exp., vol. 13, pp. 9666-9671, Nov. 2005.

[3] S. Diaz, B. Cerrolaza, G. Lasheras, and M. Lopez-Amo, "Double Raman amplified bus networks for wavelength-division multiplexing of fiber-optic sensors," J. Lightw. Technol., vol. 25, no. 3, pp. 733-739, Mar. 2007.

[4] F. T. S. Yu and S. Yin, Eds., Fiber Optic Sensors New York, Marcel Dekker, 2002.

[5] T. Y. Chang, D. S. Li, Q. M. Sui, L. Jia, Z. X. Wei, and H. L. Cui, "Applications of fiber optic Bragg grating sensing technology in a forked tunnel model," in Proc. IEEE Int. Conf. Autom. Logistics, Aug. 2007, pp. $1652-1657$.

[6] M. F. Vallejo, R. A. Perez-Herrera, C. Elosua, S. Diaz, P. Urquhart, C. Bariain, and M. Lopez-Amo, "Resilient amplified double-ring optical networks to multiplex optical fiber sensors," J. Lightw. Technol., vol. 27, no. 10, pp. 1301-1306, May 2009.

[7] E. L. Izquierdo, P. Urquhart, and M. Lopez-Amo, "Protection architectures for WDM optical fibre bus sensor arrays," J. Eng. Sci. J. Int., vol. 1, pp. 1-18, Aug. 2007.

[8] O. G. Lopez, K. Schires, P. Urquhart, N. Gueyne, and B. Duhamel, "Optical fiber bus protection network to multiplex sensors: Amplification by remotely pumped EDFAs," IEEE Trans. Instrum. Meas., vol. 58, pp. 2945-2951, Sep. 2009.

[9] P. C. Peng, H. Y. Tseng, and S. Chi, "A novel fiber-laser-based sensor network with self-healing function," IEEE Photon. Technol. Lett., vol. 15, no. 2, pp. 275-277, Feb. 2003. 
[10] L. C. G. Valente, A. M. B. Braga, A. S. Ribeiro, R. D. Regazzi, W. Ecke, C. Chojetzki, and R. Willsch, "Combined time and wavelength multiplexing technique of optical fiber grating sensor arrays using commercial OTDR equipment," IEEE Sensors J., vol. 3, no. 2, pp. 31-35, Feb. 2003.

[11] L. Yuan and L. Zhou, " $1 \times \mathrm{N}$ star coupler as a distributed fiber-optic strain sensor in a white-light interferometer," Appl. Opt., vol. 37, pp. 4168-4172, Jul. 1998.

[12] J. Montalvo, C. Vazquez, and D. S. Montero, "CWDM self-referencing sensor network based on ring resonators in reflective configuration," Opt. Exp., vol. 14, pp. 4601-4610, May 2006.

[13] L. Talaverano, S. Abad, S. Jarabo, and M. Lopez-Amo, "Multiwavelength fiber laser sources with Bragg-grating sensor multiplexing capability," J. Lightw. Technol., vol. 19, no. 4, pp. 553-558, Apr. 2001.

[14] P. C. Peng, H. Y. Tseng, and S. Chi, "A hybrid star-ring architecture for fiber Bragg grating sensor system," IEEE Photon. Technol. Lett., vol. 15, no. 9, pp. 1270-1272, Sep. 2003.

[15] C. Ragusa, A. Liotta, and G. Pavlou, "An adaptive clustering approach for the management of dynamic systems," IEEE J. Select. Areas Commun., vol. 23, no. 12, pp. 2223-2235, Dec. 2005.

[16] L. Zhang, Y. Liu, J. A. R. Williams, and I. Bennion, "Enhanced FBG strain sensing multiplexing capacity using combination of intensity and wavelength dual-coding technique," IEEE Photon. Technol. Lett., vol. 11, no. 12, pp. 1638-1640, Dec. 1999.

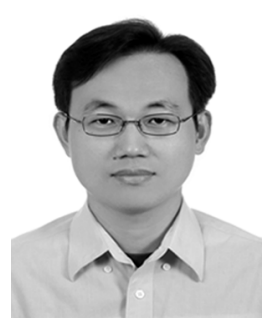

Shu-Tsung Kuo was born in Kaohsiung, Taiwan, in 1973. He received the B.S. degree and the M.S. degree in electrical engineering from the Yuan-Ze University, Taoyuan, Taiwan, in 1996 and 1999, respectively. He is currently working towards the Ph.D. degree at the Department of Electrical Engineering, National Chiao-Tung University, Hsinchu, Taiwan.

His research interests are in optical communication systems and fiber sensing networks.

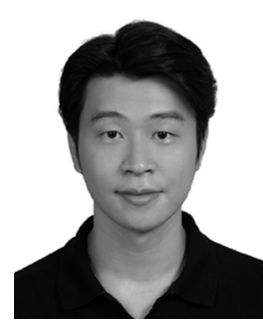

Peng-Chun Peng received the Ph.D. degree from the Institute of Electro-Optical Engineering, National Chiao Tung University, Hsinchu, Taiwan, in 2005.

From 2006 to 2008, he was an Assistant Professor with the Department of Applied Materials and Optoelectronic Engineering and the Department of Electrical Engineering, National Chi Nan University, Nantou County, Taiwan. In 2008, he joined the Department of Electro-Optical Engineering, National Taipei University of Technology, Taipei, Taiwan, as an Assistant Professor. His research interests include optical communication systems, fiber sensors, optical signal processing, and microwave photonics.

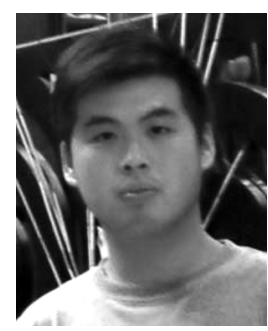

Jen-Wei Sun received the B.S. degree in electrical engineering from Huafan University, Taiwan, in 2007, and the M.S. degree in electrical engineering from National Chi Nan University, Taiwan, in 2009.

$\mathrm{He}$ is interested in optical sensor networks and WDM mesh networks.

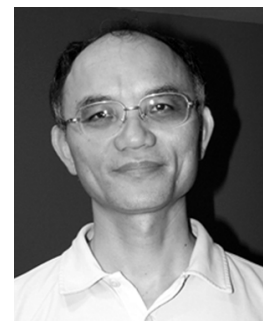

Ming-Seng Kao (S'89-M'90) was born in Taipei, Taiwan, in 1959. He received the B.S.E.E. degree from the National Taiwan University, Taipei, in 1982, the M.S. degree in optoelectronics from the National Chiao-Tung University, Hsinchu, in 1986, and the $\mathrm{Ph} . \mathrm{D}$. degree in electrical engineering from the National Taiwan University, in 1990.

From 1986 to 1987, he was an Assistant Researcher at the Telecommunications Laboratories, Chung-Li, Taiwan. In 1990, he joined the faculty of National Chiao-Tung University, where he is now a Professor of Communication Engineering. From 1993 to 1994, he was a Visiting Professor at the Swiss Federal Institute of Technology (ETH), Zurich, Switzerland, where he worked in the area of optical communications. He is currently interested in high-speed optical networks and wireless communications. 